\title{
Rab3A Mediates Vesicle Delivery at Photoreceptor Ribbon Synapses
}

\author{
Miao Tian, C. Shan Xu, Rachel Montpetit, and Richard H. Kramer \\ Department of Molecular and Cell Biology, University of California, Berkeley, Berkeley, California 94720
}

Rab3A is a synaptic vesicle-associated protein found throughout the nervous system, but its precise function is unknown. Genetic knock-out studies show that Rab3A is not necessary for vesicular release or replenishment at conventional synapses in the brain. Here we explore the function of Rab3A at ribbon synapses in the retina of the tiger salamander (Ambystoma tigrinum). Fluorescently labeled Rab3A, delivered into rods and cones through a patch pipette, binds to and dissociates from synaptic ribbons. Experiments using nonphosphorylatable GDP analogs and a GTPase-deficient Rab3A mutant indicate that ribbon binding and dissociation are governed by a GTP hydrolysis cycle. Paired recordings from presynaptic photoreceptors and postsynaptic OFF-bipolar cells show that the Rab3A mutant blocks synaptic release in an activity-dependent manner, with more frequent stimulation leading to more rapid block. The frequency dependence of block by exogenous Rab3A suggests that it acts competitively with synaptic vesicles to interfere with their resupply to release sites. Together, these findings suggest a crucial role of Rab3A in delivering vesicles to $\mathrm{Ca}^{2+}$-dependent release sites at ribbon synapses.

\section{Introduction}

Rab3A is a small G-protein belonging to the Ras superfamily of proteins that regulate membrane trafficking between different subcellular compartments in organisms from yeast to man. Despite its abundance and synaptic vesicle-specificity, the precise function of Rab3A has remained unclear (Geppert and Südhof, 1998). The Rab3A binding protein RIM is localized to the presynaptic membrane of conventional synapses (Wang et al., 1997), implicating Rab3A to RIM binding as underlying vesicle docking at the active zone. However, knock-out mice lacking Rab3A are viable and exhibit no changes in basic synaptic parameters (Geppert et al., 1994), including the kinetics of neurotransmitter release and the size of the readily releasable pool. More detailed studies have revealed effects during repeated rounds of neurotransmitter release (Geppert et al., 1997) and there are alterations in short-term (Schlüter et al., 2006) and long-term synaptic plasticity (Castillo et al., 1997; Lonart et al., 1998) that are associated with learning and memory deficits (D'Adamo et al., 2004). However, considering the prevalence of Rab3A at synapses throughout the nervous system, the Rab3A knock-out mouse has a remarkably subtle phenotype.

Interpreting the Rab3A knock-out phenotype is complicated by the existence of 3 other Rab3 isoforms (Rab3B, Rab3C, and

Received Jan. 17, 2012; revised March 27, 2012; accepted March 29, 2012.

Author contributions: M.T., C.S.X., and R.H.K. designed research; M.T., C.S.X., and R.M. performed research; M.T. and C.S.X. analyzed data; M.T. and R.H.K. wrote the paper.

This work was supported by grants from the NIH (EY-015514 and EY-003176). We thank David King for help with protein purification, Matt Welch for help with FPLC, and David Zenisek for providing Alexa-488-RBP.

Correspondence should be addressed to Richard H. Kramer, University of California, Berkeley, Department of Molecular and Cell Biology, 121 Life Sciences Addition, Berkeley, CA 94720. E-mail: rhkramer@berkeley.edu.

C. S. Xu's present address: Janelia Farm Research Campus of Howard Hughes Medical Institute, Ashburn, Virginia 20147.

DOI:10.1523/JNEUROSCI.0265-12.2012

Copyright $\odot 2012$ the authors $\quad 0270-6474 / 12 / 326931-06 \$ 15.00 / 0$
Rab3D) that may have redundant or overlapping functions. Rab3B and Rab3C are also associated with synaptic vesicles (Schlüter et al., 2002). Knock-out mice lacking two Rab3 isoforms are viable, but mice lacking three or more of the Rab3 proteins do not survive whenever Rab3A is one of the deleted proteins (Schlüter et al., 2004). Genetic deletion of one protein often results in compensatory changes in the expression levels of other functionally related proteins. Most synaptic proteins are expressed at normal levels in Rab3A knock-out mice, but at least one (rabphilin) is dramatically reduced (Geppert et al., 1994).

In this report, we have taken an alternative approach to understanding Rab3A function, by directly introducing modified versions of the Rab3A protein into presynaptic terminals. Specifically, we have investigated the presynaptic terminals of retinal rods and cones, which possess synaptic ribbons. Synaptic ribbons are proteinaceous structures that tether synaptic vesicles near release sites, supporting continuous exocytosis (Thoreson, 2007; Matthews and Fuchs, 2010). Synaptic ribbons in rods, cones, and bipolar cells extend into the cytoplasm, approximately perpendicular to the plasma membrane, far enough to accommodate several rows of synaptic vesicles. How the vesicles initially bind to the ribbon, and how they descend toward release sites at the plasma membrane is unknown. The synaptic ribbon contains RIM1, making Rab3A a candidate for mediating vesicle binding. Here we provide evidence that Rab3A reversibly binds to the synaptic ribbon in a GTPase-dependent manner. Furthermore, we show that the Rab3A binding site is necessary for the trafficking of vesicles for $\mathrm{Ca}^{2+}$-dependent exocytosis.

\section{Materials and Methods}

Plasmids. The pEGFP-rat Rab3A plasmid (a gift from Dr. Venkatesh Murthy, Harvard University, Cambridge, MA) was amplified by PCR using primers containing NheI/NotI restriction sites and inserted into 
the pET21a (+) vector to generate expression constructs for Rab3A. The membrane anchoring motif (C-terminal Cys-Ala-Cys) of the Rab3A gene was deleted using PCR to keep the protein cytosolic $($ Rab3A- $\Delta)$. Mutant Rab3A- $\Delta$ plasmid carrying a glutamine to a leucine substitution at amino acid position 81 (Q81L) was obtained by site-directed mutagenesis (Agilent Technologies). Addition of the Q-tag to the N terminus of the Rab3A gene was achieved by PCR and cloned into pET21a $(+)$ via NheI/NotI. All constructs were verified by DNA sequencing.

Protein expression and purification. Recombinant wild-type Rab3A- $\Delta$ and mutant Rab3A- $\Delta$ Q81L proteins were expressed in Escherichia coli BL21(DE3) cells. After cell lysis His-tagged proteins were purified on a nickel column (His MiniTrap, GE Healthcare). A size exclusion column (PD MiniTrap G-25, GE Healthcare) was used to further purify the proteins and to exchange the buffer solution to one containing (in $\mathrm{mM}$ ) 90 $\mathrm{CsCl}, 10 \mathrm{TEACl}, 2 \mathrm{MgCl}_{2}, 25 \mathrm{HEPES}$ and 5 EGTA (equilibration buffer). Purity and molecular weight of the proteins were confirmed by SDS-PAGE.

Fluorophore labeling. To label wild-type and mutant Rab3A- $\Delta$, we used transglutaminase-catalyzed ligation of cadaverine-functionalized Alexa647 fluorophore onto a Q-tag (PKPQQFM) fused to the $\mathrm{N}$ terminus of Rab3A (Lin and Ting, 2006). The Q-tag labeling ensures that the fluorophore attaches to Rab3A- $\Delta$ at a nonessential site. Consistent with this, the degree of labeling $([$ dye $] /[\operatorname{Rab} 3 \mathrm{~A}-\Delta])$ was close to 1 . The labeling process did not significantly change the molecular weight of Rab3A- $\Delta$. Purified Rab3A- $\Delta$ proteins were concentrated to $8 \mathrm{mg} / \mathrm{ml}$ using Amicon Ultra- 4 column (Millipore) in the equilibration buffer. The labeling reaction conditions were as follows: $150 \mu \mathrm{M}$ Rab3A- $\Delta$ proteins, $100 \mathrm{~mm}$ HEPES $\mathrm{pH} 7.4,10 \mathrm{~mm} \mathrm{CaCl}_{2}, 1 \mathrm{~mm}$ Alexa 647-cadaverine (Invitrogen), $640 \mathrm{ng} / \mu \mathrm{l}$ transglutaminase (Sigma). After a $3 \mathrm{~h}$ reaction at $4^{\circ} \mathrm{C}$, the labeled Rab3A- $\Delta$ (Fl-Rab3A- $\Delta$ ) were purified again using a gel filtration column (Superdex 75, GE Healthcare) with FPLC (AKTA, GE Healthcare) to remove free dye and transglutaminase. The purified Fl-Rab3A- $\Delta$ was concentrated again to $8 \mathrm{mg} / \mathrm{ml}$ using an Amicon Ultra-4 column and then aliquoted and stored at $-80^{\circ} \mathrm{C}$. The protein and dye concentrations were measured using the Bio-Rad Protein Assay kit and a Nanodrop (Nanodrop Technologies) respectively.

GTPase assay. The GTPase activity of purified Rab3A proteins was measured with a colorimetric assay kit (Innova Biosciences) and compared with the results of a standard curve (absorbance versus concentration of Pi). Enzyme activity is expressed as the amount ( $\mu \mathrm{mol}$ ) of GTP catalyzed by $1 \mu \mathrm{mol}$ of enzyme per min.

Retinal tissue preparation. Retinal slices from the male and female larval tiger salamander (Ambystoma tigrinum) were prepared using procedures approved by the University of California, Berkeley Institutional Animal Care and Use Committee. Salamanders were anesthetized by $0.02 \%$ Benzocaine and then killed by decapitation. The retinal slices were prepared as described previously (Rabl et al., 2006). The eyes were enucleated and the anterior segment including the lens was removed. The eyecup was cut into quarters and a section of eyecup was placed vitreal side down on a piece of filter paper $(3 \times 5$ $\mathrm{mm} ; 0.8 \mu \mathrm{m}$ pores; Millipore). The isolated retina was cut into slices $(200 \mu \mathrm{m})$ with a razor blade tissue chopper (Stoelting). These retinal slices were positioned in a recording chamber and were used for both imaging and electrophysiology.

Imaging. Laser scanning confocal microscopy of living neurons (rods and cones) was performed on the stage of an upright microscope (Zeiss LSM-510 META AxioImager), acquiring green fluorescence with a $488 \mathrm{~nm}$ argon laser and far-red fluorescence using a 633 $\mathrm{nm} \mathrm{HeNe}$ laser, sequentially, using matched pinhole sizes near 1 airy unit. The pinholes for each channel were aligned individually based upon the lightpath configuration, using multicolored subresolution bead samples. Images were acquired with Zeiss LSM 3.5 and ImageJ was used to analyze the average fluorescence of the region of interest. The cells were loaded with fluorescent proteins via a whole-cell patch pipette. For quantifying ribbon binding, the fluorescence of the FlRab3A- $\Delta$-labeled spot (i.e., the ribbon) was compared with the cytoplasm fluorescence away from the ribbons. For fluorescence recovery after photobleaching (FRAP) experiments, a bright laser spot in the terminal was scanned for five times at maximum laser power with a speed of $51.2 \mu \mathrm{s} /$ pixel. Statistical comparisons and graphing were performed using Microsoft Excel.

Measuring the number of Rab3A binding sites per ribbon. Rods were whole-cell patch-clamped and dialyzed for $10 \mathrm{~min}$ with an intracellular solution containing a concentration of $30 \mu \mathrm{M}$ Fl-Rab3A- $\Delta$ sufficient to saturate ribbon binding. The fluorescence intensity was linearly related to the concentration of Fl-Rab3A- $\Delta(10 \sim 90 \mu \mathrm{M}$; data not shown). The rod terminals were imaged using confocal microscopy, and analysis was restricted to the single labeled spot (i.e., a ribbon) that was nonoverlapping with other labeled ribbons. The intensity of a labeled spot $\left(\mathrm{F}_{\text {spot }}\right)$ was measured in a square region $(\alpha$ $\mu \mathrm{m}^{2}$ ) encompassing the spot and was compared with several other regions adjacent to the ribbon, which were used to estimate the background intensity of the cytoplasm $\left(\mathrm{F}_{\text {cyt }}\right)$ within the terminal. To convert units of fluorescence into the number of Rab3A proteins, we measured the fluorescence within a patch pipette containing $30 \mu \mathrm{M}$ Fl-Rab3A- $\Delta$ at a location where the pipette diameter approximated that of a rod terminal. At this location, the fluorescent intensity $\left(\mathrm{F}_{\mathrm{pi}}\right.$ pette) of a region $\left(\alpha \mu \mathrm{m}^{2}\right)$ was measured. To estimate the optical section thickness, we took a series of $z$-sections through a fluorescent FluoSphere bead (diameter $100 \mathrm{~nm}$, Molecular Probe) and plotted the fluorescence as a function of the vertical distance. The section thickness was determined by the full width at half maximum (FWHM) of the point spread function. The averaged optical thickness was $2 \mu \mathrm{m}$. We then obtained the volume of the recorded region, which is $\sim 2 \alpha$ $\mu \mathrm{m}^{3}$. The concentration of Fl-Rab3A- $\Delta$ in the cytoplasm of the terminal is:

$$
[\text { Fl-Rab3A- } \Delta]_{\text {cyt }}=[\text { Fl-Rab3A- } \Delta]_{\text {pipette }} \times\left(\mathrm{F}_{\text {cyt }} / \mathrm{F}_{\text {pipette }}\right)
$$

The number of Fl-Rab3A- $\Delta$ molecule in the terminal cytoplasm in $2 \alpha$ $\mu \mathrm{m}^{3}$ is:

$$
\text { \#Fl-Rab3A- } \Delta_{\text {cyt }}=[\text { Fl-Rab3A- } \Delta]_{\text {cyt }} \times 6.02 \times 10^{23} \mathrm{M}^{-1} \mathrm{~L}^{-1} \times 2 \alpha \mu \mathrm{m}^{3}
$$

The number of binding sites on the ribbon was calculated using the following equation:

$$
\# \text { Fl-Rab3A- } \Delta_{\text {ribbon }}=\# \text { Fl-Rab3A- } \Delta_{\text {cyt }} \times\left[\left(\mathrm{F}_{\text {spot }}-\mathrm{F}_{\text {cyt }}\right) / \mathrm{F}_{\text {cyt }}\right]
$$

The surface area of the rod ribbon is $\sim 4 \mu \mathrm{m}^{2}$ (Thoreson et al., 2004), so the density of Fl-Rab3A- $\Delta$ on the ribbon is \# Fl-Rab3A- $\Delta_{\text {ribbon }} / 4 \mu \mathrm{m}^{2}$.

Electrophysiology. For whole-cell patch clamp, retinal slices were superfused at $\sim 1 \mathrm{ml} / \mathrm{min}$ with extracellular solution containing the following (in mM): $114 \mathrm{NaCl}, 2.5 \mathrm{KCl}, 2 \mathrm{CaCl}_{2}, 0.5 \mathrm{MgCl}_{2}, 10 \mathrm{HEPES}$, and 5 Glucose, pH 7.8 (Rabl et al., 2006). To isolate rod $\mathrm{Ca}^{2+}$ currents, $1 \mathrm{~mm}$ TEA, $200 \mathrm{~nm}$ charybdotoxin (CbTX), and $100 \mu \mathrm{M}$ niflumic acid were added to block $\mathrm{K}^{+}, \mathrm{Ca}^{2+}$-dependent $\mathrm{K}^{+}$, and $\mathrm{Ca}^{2+}$-dependent $\mathrm{Cl}^{-}$currents, respectively. Paired whole-cell voltage-clamp recordings were obtained from rods/cones and OFF bipolar cells using $7 \sim 15$ $\mathrm{M} \Omega$ patch pipettes pulled from borosilicate glass. The pipette solution contained (in mM) $90 \mathrm{CsOH}, 90$ gluconate acid, 10 TEACl, 2 $\mathrm{MgCl}_{2}, 10$ HEPES, 5 EGTA, $10 \mathrm{MgATP}$ and 0.5 GTP-tris, $\mathrm{pH}$ 7.2. Rods and cones were voltage clamped to $-70 \mathrm{mV}$, bipolar cells to $-50 \mathrm{mV}$, using a Multiclamp amplifier (700B, Axon Instruments). Currents were acquired using a Digidata 1440 interface and pClamp 10 software (Molecular Devices). Recordings in which the access resistance was $>40 \mathrm{M} \Omega$ were rejected. The criterion for statistical significance was chosen to be $p<0.05$ and evaluated with the Student's $t$ tests. Variability is reported as \pm SEM.

\section{Results}

To investigate whether Rab3A could mediate vesicle binding to the synaptic ribbon, we expressed in E. coli a recombinant version of Rab3A, which after purification was tagged with a small-molecule fluorophore (Fig. $1 A$ ). We deleted the normal $\mathrm{C}$ terminus of Rab3A, removing the lipidation motif required for vesicle membrane anchoring (Johnston et al., 1991). The Rab3A construct was also modified on the $\mathrm{N}$ terminus to in- 
A

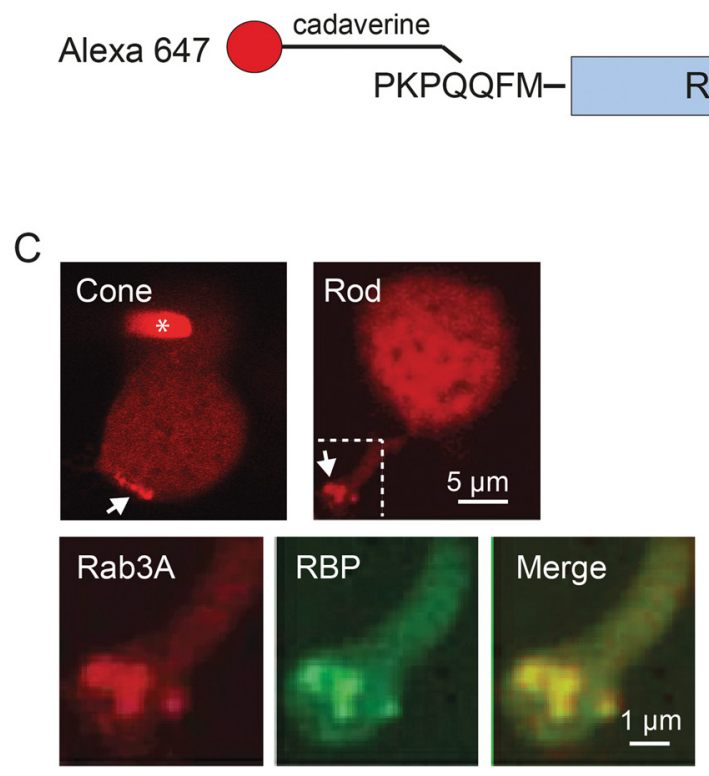

B

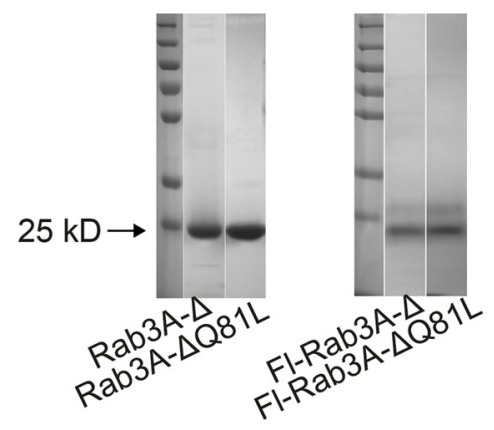

E

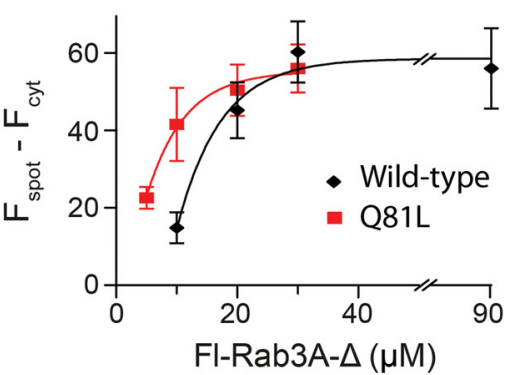

Figure 1. FI-Rab3A- $\Delta$ binds to synaptic ribbons. A, A schematic diagram of the fluorescent Rab3A probe. The glutamine side chain of the N-terminal Q-tag (PKPQQFM) was conjugated to the polyamine-containing probe Alexa 647 -cadaverine by transglutaminase-catalyzed ligation. A His-tag was fused to the $C$ terminus for protein purification. $\boldsymbol{B}$, Purity of the protein preparations for both the wild-type and Q81L Rab3A-probes was established by SDS-PAGE Gel electrophoresis, showing the majority of the proteins was in a dark band at the expected molecular weight (25 kDa). C, Top, Dialysis of Fl-Rab3A- $\Delta$ through a patch pipette into a cone (left) or into a rod (right) results in accumulation at ribbons in the synaptic terminals (arrows). The asterisk in the cone shows the patch pipette. Bottom, A magnified view of the synaptic terminal region of the rod. Spots labeled with FI-Rab3A- $\Delta$ (red) colocalize with spots labeled with Alexa-488-RBP (green). $\boldsymbol{D}$, Independent photobleaching indicates colocalization is not an artifact of spectral overlap between fluorophores. Fl-Rab3A- $\Delta$ localized on the ribbon could be photobleached repeatedly with 633 nm light (arrows) without affecting Alexa-488-RBP. E, The ribbon fluorescence with different concentrations of FI-Rab3A- $\Delta$ in the pipette.

clude a Q-tag labeling domain (PKPQQFM), which enabled enzymatic conjugation to a cadaverine-containing fluorophore derivative (Lin and Ting, 2006). Finally, a His-tag was added to the $\mathrm{N}$ terminus for affinity purification on a $\mathrm{Ni}^{2+}$ column. PAGE showed a single major band both before and after fluorophore labeling (Fig. $1 B$ ). The purified protein, which we named Fl-Rab3A- $\Delta$, was soluble up to at least 400 $\mu \mathrm{M}$ in physiological buffer.

The purified Fl-Rab3A- $\Delta$ protein was introduced through a patch pipette into giant rod and cone photoreceptors of the tiger salamander. Fl-Rab3A- $\Delta$ accumulated in spots at the base of the synaptic terminals of these cells, colocalizing with structures labeled with Alexa-488-tagged RIBEYE binding peptide (Alexa488-RBP) (Fig. 1C), a marker of the synaptic ribbon (Zenisek et al., 2004). Colocalization of the two proteins was observed in each of the 109 presumptive ribbons in a total of 22 rods examined. Selective excitation of Fl-Rab3A- $\Delta$ resulted in photobleaching without affecting Alexa-488-RBP fluorescence (Fig. 1D). The independent photobleaching confirms that colocalization of the two proteins was not an artifact of spectral overlap between their fluorophores, but rather represents binding to a common structure. Since RBP binds specifically to synaptic ribbons (Zenisek et al., 2004), these findings strongly suggest that Rab3A also binds to the ribbons.

We estimated the density of Rab3A binding sites on the ribbon by determining the amount of Fl-Rab3A- $\Delta$ that is required to saturate labeling (Fig. $1 E$ ). Using the fluorescence intensity of the pipette as a reference for dye concentration, we estimated the density to be $3077 \pm 352$ molecules $/ \mu \mathrm{m}^{2}(n=5)$, far exceeding the number of vesicles on a fully packed synaptic ribbon $(\sim 400 /$ $\mu \mathrm{m}^{2}$; Thoreson et al., 2004). This indicates that there are sufficient binding sites to accommodate at least one Rab3A from every tethered vesicle.
A

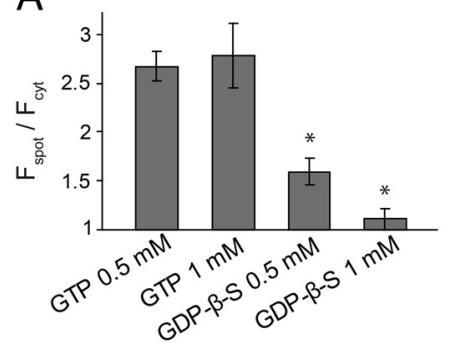

C
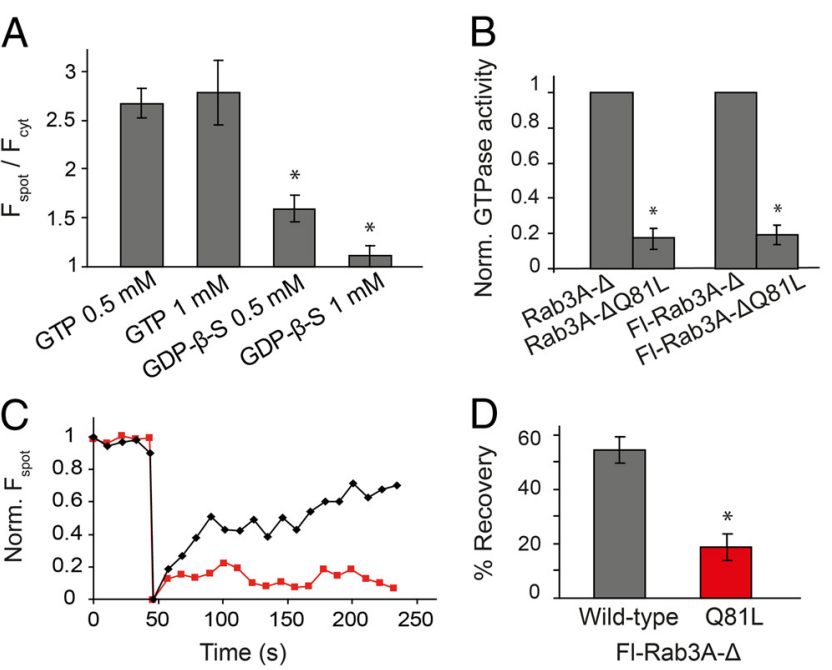

Figure 2. Fl-Rab3A- $\Delta$ binding to ribbons is GTP-dependent. $\boldsymbol{A}$, Ribbon labeling requires GTP. GDP- $\beta$-S decreased FI-Rab3A- $\Delta$ labeling versus GTP (1 mm). ${ }^{*} p<0.01$ ( $\left.n=4 \sim 17\right)$. $\boldsymbol{B}$, GTP hydrolysis assay showing that the Q81L mutant had significantly less GTPase activity $(<20 \%)$ than the wild-type protein $(n=4),{ }^{*} p<0.01$. C, FRAP of wild-type (black) and Q81L (red) $\mathrm{Fl}-\mathrm{Rab} 3 \mathrm{~A}-\Delta$ on the ribbon indicates that GTP hydrolysis is required for Rab3A dissociation from the ribbon. The patch pipette contained $30 \mu \mathrm{m}$ of either protein in these experiments. $\boldsymbol{D}$, Quantification of FRAP results. Mean FRAP is decreased with the $081 \mathrm{~L}$ mutant compared with wild type. ${ }^{*} p<0.01(n=4 \sim 5)$.

Rab3A cycles between active GTP-bound and inactive GDP-bound forms, but only the GTP-bound form binds to RIM (Geppert and Südhof, 1998). We found that ribbon labeling by Fl-Rab3A- $\Delta$ occurred when the pipette contained GTP, but not GDP- $\beta$-S, a nonphosphorylatable GDP analog (Fig. 2A). Rab3A can be locked into a GTP-bound active con- 
A

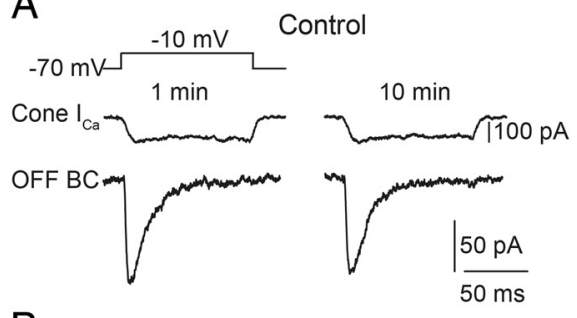

B

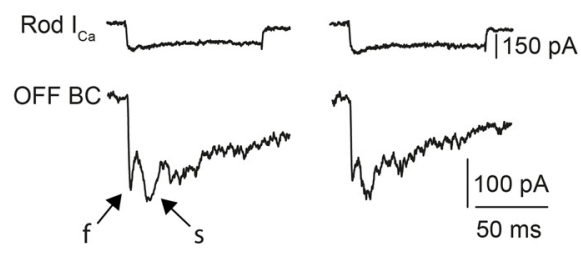

FI-Rab3A- $\triangle Q 81 L$
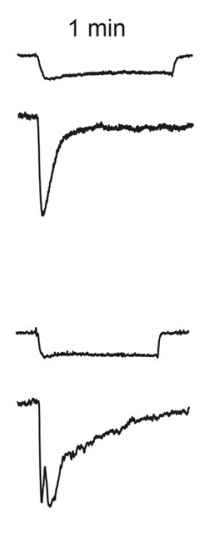
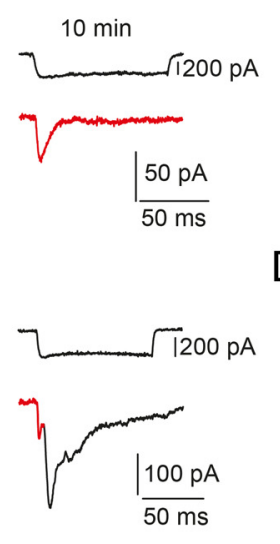
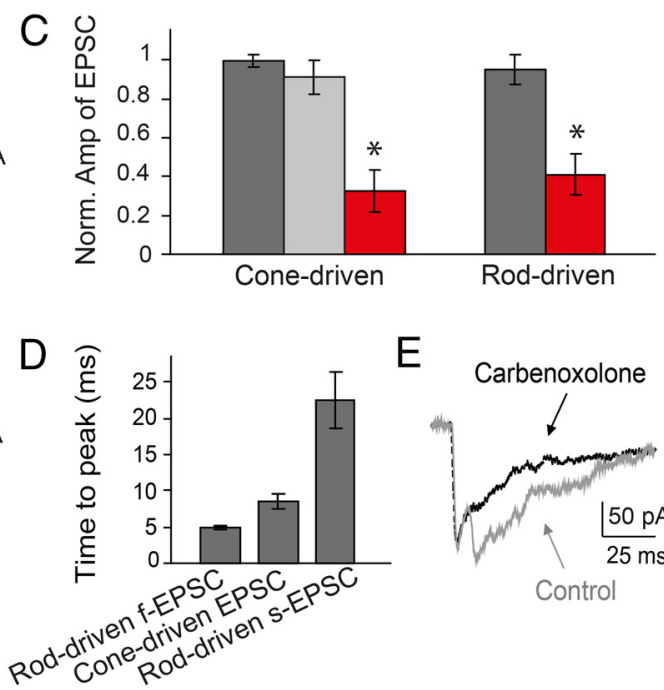

$\mathrm{E}$

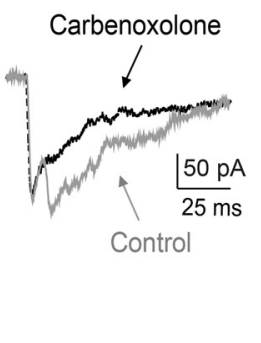

Figure 3. Rab3A- $\triangle Q 81 \mathrm{~L}$ blocks synaptic release from rods and cones. $A, B$, Cone-driven EPSCs and rod-driven f-EPSCs decreased little over 10 min in control experiments (no protein in pipette) but decreased significantly with Rab3A- $\Delta$ Q81L in the pipette $(20 \mu \mathrm{M})$. C, Quantification of blockade of synaptic release by the mutant Rab3A. EPSC amplitude with no protein (gray), wild-type Rab3A- $\Delta$ (light gray), or Rab3A- $\Delta$ Q81L (red). EPSCs were measured at 10 min after break-in, $n=3 \sim 5$; ${ }^{*} p<0.01$ versus (ontrol (gray). D, Rod-driven EPSPs have two kinetic components. Following the onset of a depolarizing pulse in the rod, the time-to-peak of the resulting s-EPSC was $22.4 \pm 3.9 \mathrm{~ms}(n=15)$, much slower than f-EPSCs (5.03 $\pm 0.3 \mathrm{~ms}, n=15)$ or cone-driven EPSC $(8.5 \pm 0.9 \mathrm{~ms}, n=8)$. $\boldsymbol{E}$, The gap-junction uncoupler carbenoxolone $(200 \mu \mathrm{M})$ selectively suppressed the rod-driven s-EPSC and had little effect on the f-EPSC, providing evidence that rod-driven $\mathrm{s}$-EPSCs are a consequence of neurotransmitter release from electrically coupled neighboring rods.

figuration by mutating the GTPase active site (the Q81L mutation; Brondyk et al., 1993). GTPase assays confirmed that both the unlabeled and labeled forms of Rab3A- $\Delta$ Q81L had $<20 \%$ of the activity of the wild-type protein (Fig. $2 \mathrm{~B}$ ). Introducing the Q81L mutation into Fl-Rab3A- $\Delta$ did not disrupt the initial binding to ribbons $\left(\mathrm{F}_{\text {spot }} / \mathrm{F}_{\text {cyt }}\right.$ of mutant $=2.4 \mathrm{vs}$ wild-type $=2.7, p>0.05)$, but it dramatically reduced the turnover of Rab3A binding, as evidenced by a decrease in FRAP on the ribbon compared with the wild-type protein (mean FRAP $=55 \%$ for wild-type vs $19 \%$ for the Q81L mutant; Fig. $2 C, D)$. The slower dissociation rate of the $\mathrm{Q} 81 \mathrm{~L}$ mutant results in the protein having a higher apparent affinity for the synaptic ribbon (Fig. $1 E$ ). FRAP of wild-type and mutant protein in small cytoplasmic regions was fast $(<0.1 \mathrm{~s})$ indicating that both proteins are freely diffusible and have a high mobility when they are unbound. These results indicate that GTPase activity is necessary for bound Rab3A to exchange with free Rab3A in the cytoplasm, suggesting that binding and dissociation is mediated by a GTP-GDP cycle. Incomplete FRAP on ribbons labeled with wild-type Rab3A- $\Delta$ may result from photodamage of the ribbon machinery, as shown previously with fluorophore-assisted light inactivation via labeled RBP (Snellman et al., 2011).

To determine whether Rab3A binding is essential for synaptic transmission, we obtained paired recordings from a presynaptic photoreceptor and a postsynaptic OFF bipolar cell in a retinal slice. Photoreceptors were depolarized from -70 to $-10 \mathrm{mV}$ to open voltage-gated $\mathrm{Ca}^{2+}$ channels and trigger enough $\mathrm{Ca}^{2+}$ dependent exocytosis to empty the readily releasable pool (Rabl et al., 2006). The depolarizing stimulus was repeated at $30 \mathrm{~s}$ intervals, sufficient for complete refilling of the pool. Over $10 \mathrm{~min}$ of recording, cone-driven EPSCs decreased little when wild-type Rab3A- $\Delta$ or no protein at all was included in the patch pipette $(<10 \%)$ (Fig. $3 A, C)$. However, when Rab3A- $\Delta$ Q81L was included in the pipette the EPSCs diminished by $\sim 67 \%$ within 10 $\min$. The voltage-gated $\mathrm{Ca}^{2+}$ current was unaffected by Rab3A$\Delta \mathrm{Q} 81 \mathrm{~L}$. Hence Rab3A- $\Delta$ Q81L must suppress some step in the synaptic vesicle delivery or release process.

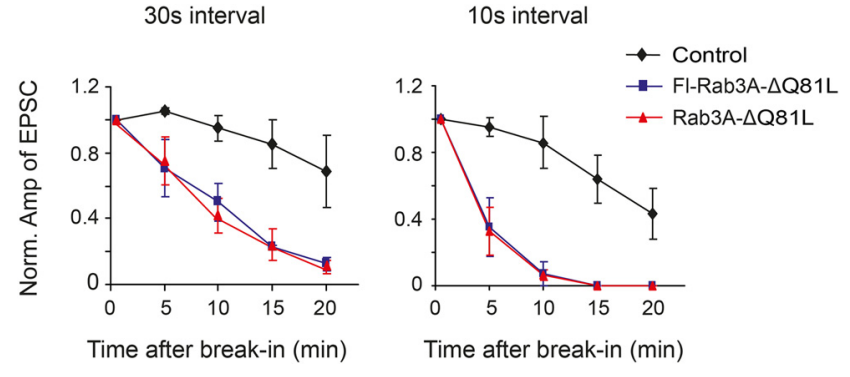

Figure 4. Rab3 A binding sites are necessary for synaptic vesicle replenishment. Blockade of synaptic release is activity-dependent, suggesting that endogenous Rab3 A on synaptic vesicles compete with exogenous Rab3A- $\Delta$ Q81L for a common binding site. Rab3A- $\Delta$ Q81L suppression of rod-driven $\mathrm{f}$-EPSCs occurred faster with a higher rate of stimulation $(n=3 \sim 5)$.

Rod-driven EPSCs had a fast component (f-EPSC) similar to cone-driven EPSCs, but also a secondary slower component (sEPSC; Fig. $3 B, D$ ). Inclusion of Rab3A- $\Delta \mathrm{Q} 81 \mathrm{~L}$ in the rod patch pipette reduced the f-EPSC by $\sim 59 \%$, but had no effect on the s-EPSC (Fig. 3B,C). The f-EPSC appears to be mediated by a direct monosynaptic connection from the rod to the bipolar cell whereas the s-EPSC is mediated by electrically coupled rods with synaptic contacts onto the same bipolar cell. Because the Rab3A$\Delta \mathrm{Q} 81 \mathrm{~L}$ cannot cross gap junctions, it reduces synaptic release only from the rod dialyzed by the patch pipette, hence selectively reducing the f-EPSC. Consistent with this explanation, the gap junction uncoupler carbenoxolone had little effect on the f-EPSC, but it blocked the s-EPSC (Fig. 3E), consistent with previous observations (Cadetti et al., 2005).

If Rab3A binding to the ribbon is a necessary step in synaptic release, exogenous Rab3A- $\Delta$ should compete with endogenous Rab3A in inhibiting release. Furthermore, Rab3A binding sites should be more frequently vacated when the vesicle release rate is faster, because there will be more opportunities for Rab3A to bind. We tested these predictions by varying the stimulation frequency during Rab3A- $\Delta$ Q81L dialysis into rods. With a $10 \mathrm{~s}$ interval between depolarizing stimuli, EPSCs were suppressed 
approximately twice as fast as with a $30 \mathrm{~s}$ interval (Fig. 4). Hence while Rab3A- $\Delta$ Q81L has little effect on vesicles that are already docked and primed for release, it suppresses the delivery of new vesicles into the immediately releasable pool.

\section{Discussion}

Ribbon synapses are specialized for transmitting information about graded sensory signals whereas conventional synapses are specialized for transmitting information about action potentials. To operate differently, the two types of synapses must contain different proteins, or contain common proteins that carry out different tasks. Vesicles at conventional synapses possess synap$\sin$, an actin-binding protein that tethers vesicles to the cytoskeleton, accounting for the "reserve pool" of immobilized vesicles (Hilfiker et al., 1999). There is no synapsin in ribbon synapses (Mandell et al., 1990), so synaptic vesicles are freely diffusible (Holt et al., 2004; Rea et al., 2004). Instead of having a reserve pool, several lines of evidence support the view that the synaptic ribbon serves as a necessary way-station that binds vesicles and regulates how many are available for release. Mutations that cause detachment of synaptic ribbons disrupt synaptic transmission from rods and cones (Dick et al., 2003). Recent studies using fluorophore-assisted laser inactivation of RIBEYE implicate the ribbon as necessary for supplying vesicles for release in cones and bipolar cells (Snellman et al., 2011). Finally, in cones, the base of the synaptic ribbon is depleted of synaptic vesicles in darkness, quantitatively accounting for the diminished size of the readily releasable pool (Jackman et al., 2009).

Our results indicate that Rab3A is crucial for ribbon-mediated delivery of vesicles. The Rab3A binding proteins RIM1 and RIM2 are both expressed in photoreceptor terminals, but immunogold EM studies show that only RIM1 is located on the synaptic ribbon. RIM2 is found adjacent to the base of the ribbon, but exclusively on the plasma membrane (tom Dieck et al., 2005). Hence it is likely that RIM1 serves as the site on the ribbon for Rab3Aribbon binding, and therefore synaptic vesicle attachment. Our results show that the binding and unbinding of Rab3A to the ribbon are dependent on GTPase activity. The association and dissociation of Rab3A from RIM involves a GDP/GTP cycle, so we propose that each vesicle attachment event corresponds to one round of GTPase activity. GTP-bound Rab3A would escort vesicles to the ribbon during the initial attachment. Other proteins may facilitate dissociation of Rab3A from the vesicle (Geppert and Südhof, 1998), returning Rab3A to the cytoplasm where it can encounter and escort another vesicle.

We found that the faster vesicles are exocytosed by repeating depolarizations, the faster exogenous Rab3A- $\Delta$ Q81L interferes with the resupply of vesicles. The activity-dependence of blockade implies that exogenous Rab3A- $\Delta \mathrm{Q} 81 \mathrm{~L}$ competes with endogenous synaptic vesicles for a common binding site on the ribbon. This binding site may mediate only the initial attachment of vesicles to the ribbon, but it could also mediate movement of vesicles along the ribbon, toward the membrane. In this scenario, vesicles would undergo a series of Rab3A-mediated binding events, as suggested by the presence of multiple RIM1 clusters along the length of the ribbon (tom Dieck et al., 2005). Further experiments, perhaps involving visualization of single synaptic vesicles, are necessary to characterize how vesicles move along the ribbon.

Deleting Rab3A has no effect on the $\mathrm{Ca}^{2+}$-dependent release rate or on the replenishment rate at conventional synapses, but it does alter the amount of neurotransmitter released, which is especially noticeable during rapidly repeated stimuli. This has led to the proposal that Rab3A regulates only a late step in the vesicle fusion process, after the $\mathrm{Ca}^{2+}$ sensor is activated (Geppert et al., 1997). In conventional synapses, RIM proteins (RIM1 and RIM2) and SNARE proteins are all located in close proximity to one another at the presynaptic membrane, providing a direct opportunity for Rab3A to regulate fusion. At ribbon synapses, RIM1 molecules are on the ribbon, spatially segregated from proteins mediating fusion. This difference may explain why Rab3A regulates vesicle delivery in ribbon synapses, but not in conventional synapses.

\section{References}

Brondyk WH, McKiernan CJ, Burstein ES, Macara IG (1993) Mutants of Rab3A analogous to Oncogenic Ras mutants. J Biol Chem 268:9410-9415.

Cadetti L, Tranchina D, Thoreson WB (2005) A comparison of release kinetics and glutamate receptor properties in shaping rod-cone differences in EPSC kinetics in the salamander retina. J Physiol 569:773-788.

Castillo PE, Janz R, Südhof TC, Tzounopoulos T, Malenka RC, Nicoll RA (1997) Rab3A is essential for mossy fibre long-term potentiation in the hippocampus. Nature 388:590-593.

D’Adamo P, Wolfer DP, Kopp C, Tobler I, Toniolo D, Lipp HP (2004) Mice deficient for the synaptic vesicle protein Rab3A show impaired spatial reversal learning and increased explorative activity but none of the behavioral changes shown by mice deficient for the Rab3A regulator Gdil. Eur J Neurosci 19:1895-1905.

Dick O, tom Dieck S, Altrock WD, Ammermüller J, Weiler R, Garner CC, Gundelfinger ED, Brandstätter JH (2003) The presynaptic active zone protein bassoon is essential for photoreceptor ribbon synapse formation in the retina. Neuron 37:775-786.

Geppert M, Südhof TC (1998) Rab3 and synaptotagmin: the yin and yang of synaptic membrane fusion. Annu Rev Neurosci 21:75-95.

Geppert M, Bolshakov VY, Siegelbaum SA, Takei K, De Camilli P, Hammer RE, Südhof TC (1994) The role of Rab3A in neurotransmitter release. Nature 369:493-497.

Geppert M, Goda Y, Stevens CF, Südhof TC (1997) The small GTP-binding protein Rab3A regulates a late step in synaptic vesicle fusion. Nature 387:810-814

Hilfiker S, Pieribone VA, Czernik AJ, Kao HT, Augustine GJ, Greengard P (1999) Synapsins as regulators of neurotransmitter release. Philos Trans R Soc Lond B Biol Sci 354:269-279.

Holt M, Cooke A, Neef A, Lagnado L (2004) High mobility of vesicles supports continuous exocytosis at a ribbon synapse. Curr Biol 14:173-183.

Jackman SL, Choi SY, Thoreson WB, Rabl K, Bartoletti TM, Kramer RH (2009) Role of the synaptic ribbon in transmitting the cone light response. Nat Neurosci 12:303-310.

Johnston PA, Archer BT 3rd, Robinson K, Mignery GA, Jahn R, Südhof TC (1991) Rab3A attachment to the synaptic vesicle membrane mediated by a conserved polyisoprenylayted carboxy-terminal sequence. Neuron 7:101-109.

Lin CW, Ting AY (2006) Transglutaminase-catalyzed site-specific conjugation of small-molecule probes to proteins in vitro and on the surface of living cells. J Am Chem Soc 128:4542-4543.

Lonart G, Janz R, Johnson KM, Südhof TC (1998) Mechanism of action of Rab3A in mossy fiber LTP. Neuron 21:1141-1150.

Mandell JW, Townes-Anderson E, Czernik AJ, Cameron R, Greengard P, De Camilli P (1990) Synapsins in the vertebrate retina: absence from ribbon synapses and heterogeneous distribution among conventional synapses. Neuron 5:19-33.

Matthews G, Fuchs P (2010) The diverse roles of ribbon synapses in sensory neurotransmission. Nat Rev Neurosci 11:812-822.

Rabl K, Cadetti L, Thoreson WB (2006) Paired-pulse depression at photoreceptor synapses. J Neurosci 26:2555-2563.

Rea R, Li J, Dharia A, Levitan ES, Sterling P, Kramer RH (2004) Streamlined synaptic vesicle cycle in cone photoreceptor terminals. Neuron 41:755-766.

Schlüter OM, Khvotchev M, Jahn R, Südhof TC (2002) Localization versus function of Rab3 proteins. Evidence for a common regulatory role in controlling fusion. J Biol Chem 277:40919-40929.

Schlüter OM, Schmitz F, Jahn R, Rosenmund C, Südhof TC (2004) A com- 
plete genetic analysis of neuronal Rab3 function. J Neurosci 24:6629-6637.

Schlüter OM, Basu J, Südhof TC, Rosenmund C (2006) Rab3 superprimes synaptic vesicles for release: implications for short-term synaptic plasticity. J Neurosci 26:1239-1246.

Snellman J, Mehta B, Babai N, Bartoletti TM, Akmentin W, Francis A, Matthews G, Thoreson W, Zenisek D (2011) Acute destruction of the synaptic ribbon reveals a role for the ribbon in vesicle priming. Nat Neurosci 14:1135-1141.

Thoreson WB (2007) Kinetics of synaptic transmission at ribbon synapses of rods and cones. Mol Neurobiol 36:205-223.

Thoreson WB, Rabl K, Townes-Anderson E, Heidelberger R (2004) A highly
$\mathrm{Ca} 2+$-sensitive pool of vesicles contributes to linearity at the rod photoreceptor ribbon synapse. Neuron 42:595-605.

tom Dieck S, Altrock WD, Kessels MM, Qualmann B, Regus H, Brauner D, Fejtová A, Bracko O, Gundelfinger ED, Brandstätter JH (2005) Molecular dissection of the photoreceptor ribbon synapse: physical interaction of Bassoon and RIBEYE is essential for the assembly of the ribbon complex. J Cell Biol 168:825-836.

Wang Y, Okamoto M, Schmitz F, Hofmann K, Südhof TC (1997) Rim is a putative Rab3A effector in regulating synaptic-vesicle fusion. Nature 388:593-598

Zenisek D, Horst NK, Merrifield C, Sterling P, Matthews G (2004) Visualizing synaptic ribbons in the living cell. J Neurosci 24:9752-9759. 